\title{
Thin Film Condensation in a Tesla Turbine
}

\author{
Roberto Lisker*, Udo Hellwig, Franz Xaver Wildenauer
}

\section{Zusammenfassung}

Im Folgenden wird der Einsatz eines dünnen Flüssigkeitsfilms in der Auslassregion einer Scheibenläuferturbine beschrieben. Die zunehmenden Relativgeschwindigkeiten am Auslass, die durch die Expansion des Arbeitsmediums bei gleichzeitiger Verminderung des durchströmten Querschnittes hervorgerufen wird, führen zu einer Reduktion der Turbineneffizienz und einem höheren Treibstoffverbrauch. Um dies zu unterbinden, soll ein Teil des gasförmigen Arbeitsmediums kondensiert werden, indem sich ein laminarer, dünner Flüssigkeitsfilm bildet. In dem Flüssigkeitsfilm treten höhere Scherkräfte auf, die die Kraftübertragung verbessern. Durch die großen Unterschiede der spezifischen Volumina in der Gas- und Flüssigphase reduziert sich durch die Kondensation der Gasvolumenstrom und somit die Relativgeschwindigkeiten, was zu einer höheren Turbineneffizienz führt.

\section{Abstract}

This article deals with the development of a laminar thin film at the outlet area of a disk turbine. The increasing relative velocities at the outlet -caused by the expansion of the working fluid and the reduction of the cross- section area- are reducing the turbine efficiency, resulting in a lower power output and a higher fuel consumption. To overcome this, a certain amount of the working fluid shall condensate at the disk wall propagating a laminar thin film. Within the liquid film higher shear forces will be transported and therefore the power transfer to the disk optimized. Furthermore, and most important, due to the differences in the specific volume between the liquid and the gaseous phase, the volume flow of the gaseous phase is reduced and therefore the relative velocities are effectively reduced, resulting in a higher turbine efficiency.

\section{Introduction}

Since 2008 the Tesla turbine is a constant research topic at the Technical University of applied Sciences Wildau with the aim to improve the turbine efficiency, starting with the determination of the velocity field inside of the Tesla turbine (Lisker 2009) over various investigations to componentry (Holz 2014), and numerous computational simulations (Meller 2013). The aim is to improve the turbine efficiency, which resulted in the development of a test rig and a turbine Tesla stream turbine prototype (Lisker et al. 2015). The more recent idea to improve the turbine efficiency is the idea to apply condensation at the outlet region to prevent high tangential and radial velocities, which arise due to continuity reasons.

The Tesla turbine is due to its unconventional way to produce power a unique turbine. Invented by Nicola Tesla in 1909 (Tesla 1911), the tur- bine was never a commercial success and is regarded as a "lost invention" today. Although the fail as a commercial product, the Tesla turbine has stayed an object of academic research throughout the last decades due to its simplicity, low production costs and unique flow field. The Turbine consists of several disks, which are plane parallel arranged on a shaft. The gap between these disks is depending on the working fluid which enters the gap at the outer perimeter with a high tangential velocity. Then the fluid continues its way in a nearly spiral pathway to the exit, located at the centre of the disks. Due to frictional forces, caused by the viscosity of the working fluid, the rotor starts spinning and torque is produced. In Fig. 1 left, a Tesla turbine model is displayed. However, the continuous expansion of the working fluid in the gap element and the decreasing of the cross section whilst increasing the specific volume of the working fluid, leads to an increase in the tan- gential and radial velocities at the outlet region, causing high energetic losses. Nevertheless, the unconventional build-up of the turbine has a lot of advantages. It is resistant against particles enclosed in the working fluid stream as long as the particle diameter is smaller than the gap width. The turbine can easily be adjusted to different working fluids and conditions by simply changing the gap width. On the downside of this turbine is the limited amount of enthalpy depletion. Like all radial turbines, the maximum enthalpy depletion is determined by the diameter of the turbine rotor which is dependent on the rotational speed and the maximum tensile strain of the used material. If this threshold is reached, the working fluid has to leave the turbine for further expansion into another turbine.

Due to the high surface area in contact with the working fluid and the ability to handle particle laden flows, the turbine is predestined for the use as a heat exchanger. There are two 
possible applications. In the first application case, the turbine can be operated as high temperature gas turbine, which cools the hot gas down to a temperature level which most bladed turbines are capable of so the limited enthalpy depletion is not an issue anymore. The second application is the utilization as condensation turbine behind the main turbine. Here the working fluid can condense which reduces the size of the condenser and therefore increases the cycle efficiency. Within bladed turbines condensation is highly unwanted, due to the damage water particles or particles in general do to turbine blades. But the advantage of an opportunity to condense in a turbine is vast. The pressure can be lowered, the condenser itself can be smaller and the efficiency of the cycle will be increased. Therefore, a condensation stage benefits the cycle. By cooling the disks in the outlet area, the temperature and the specific volume of the working fluid as well as the energetic losses can be reduced. If the temperature at the outlet region is below the saturation temperature, condensation takes place. Hereby the gaseous working fluid changes its state and becomes liquid. The advantage is a much lower specific volume and thus leads to lower tangential and radial velocities. For example, the specific volume of steam at the saturation pressure of 1 bar is $1,694 \mathrm{~m}^{3} \mathrm{~kg}^{-1}$ whereas the specific volume of water at the same pressure is $0,001 \mathrm{~m}^{3} \mathrm{~kg}^{-1}$, which is 1694 times lower than the specific volume of steam.

\section{The flow field inside of a Tesla turbine gap}

In the following the flow field inside of a Tesla turbine is explained. As depicted in Fig. 1 right, the course of the Tesla turbine are the increasing relative velocities towards the outlet. The reason is the reduction of the cross- sectional area. To overcome this part of the gaseous working fluid shall condense which leads to a reduction of the tangential and radial velocities. In the following a model is presented for the determination of the radial and tangential velocities. This model is only valid for a laminar flow, which can be easily adjusted by the gap width.
1 Shaft 2 Multiple disks/rotor 3 Spacer with gap outlet 4 Housing 5 Inlet 6 Turbine Outlet

Relative Velocity: Magnitude (m/s)
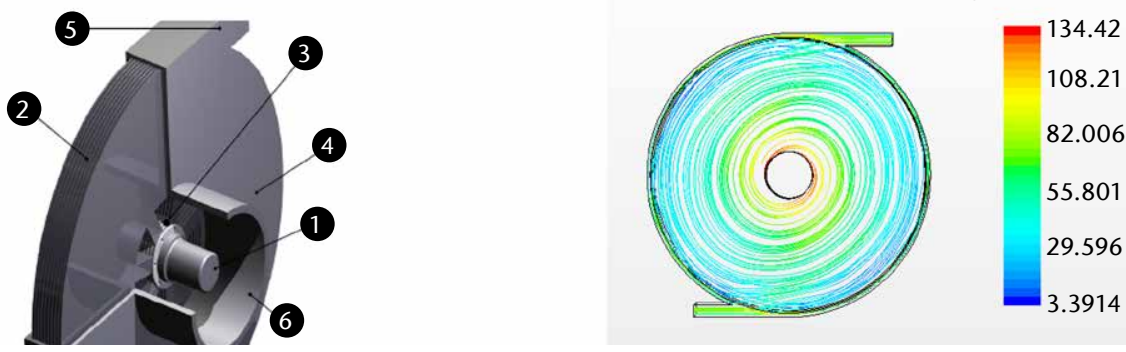

Fig. 1) Left: model of a Tesla turbine, Right: flow field inside of a Tesla turbine

\subsection{Velocity field}

The determination of the velocity field is essential for all calculations regarding design of a Tesla turbine. Especially the relative velocity between the disk and the fluid stream are important for heat transfer calculations and the determination of the propagation of the fluid film. For the determination of the velocity field it is assumed that the flow is laminar and the velocity profile has a parabolic shape. The laminar condition can be adjusted by the width of the gap $b$ and the correctness of a parabolic velocity profile has been proven by Guha and Sengupta (2013). The velocities for a laminar flow are derived from the continuity and the momentum equations. A detailed derivation was presented by Lisker (2013).

The radial velocity is in a Tesla turbine is determined by the ratio of the fluid mass flow and the cross- section area as shown in equation 1.

$c_{r}(r)=\frac{m_{m} \phi}{2 \pi b r \rho_{G}}$

Here $\mathrm{b}$ is the width of the gap, $r$ the radius, $\stackrel{m}{m} \phi$ the mass flow and $\rho_{G}$ the density of the used working fluid. From equation 1 it becomes immediately apparent, that with a reduction of the cross- section area $2 \pi b r$ the radial velocity increases. The tangential velocity is the sum of the disk velocity $r \omega$ and the relative velocity $c_{r}$. The later one can be determined according to equation 2 .

$c_{v}(r)=\frac{2 r_{1}^{2} \omega}{r \zeta}+\left[\frac{u_{1} r_{1}}{r}-\frac{2 r_{1}^{2} \omega}{r \zeta}\right] e^{\left(-\zeta \frac{1-\left(\frac{r}{r_{1}}\right)^{2}}{2}\right)}$

Here $\omega$ is the angular velocity of the disk, $r_{1}$ the outer radius, $u_{1}$ the tangential velocity at the inlet and $\zeta$ the friction coefficient for a laminar flow which stores information's about the viscosity and the radial velocity. The later one is of great importance. High viscous fluids are known to work best with the turbine. On the other hand, higher viscous working fluids can lead to a power transmission loss, due to the lack of energy transfer inside of the fluid and the resulting non-adaption of velocity changes (Lisker 2013). Due to the information's stored inside of that factor, changes in the radial velocity do affect the tangential velocity and vice versa. For the heat transfer determination, the absolute velocity c will be used to calculate the heat transferred. In the following Fig. 2 the normalised velocities are shown. The yellow line represents the relative tangential velocity ct and the grey one the radial velocity $\mathrm{cr}$. It can be seen that the velocities are rising towards the outlet at the centre of the disk. The higher the relative velocities compared to the disk velocity (blue line) the greater the efficiency losses. The ideal relative tangen- 
tial velocity would be just over the disk velocity (red dashed line), applying just enough drag that the system rotates with the load.

From the equations above some important statements regarding the operation and the performance of the turbine can be made. One can see from the radial velocity, that the impact of the mass flow is vast. A low mass flow rate reinforces the impact of the tangential velocity and the pathway of a fluid particle/element inside the gap becomes spiral shaped (Fig. 1 right). Within this mode of operation most of the kinetic energy of the working fluid is transferred to the disks. The turbine operates at its ideal state. A high mass flow rate leads to an increasing radial velocity and the tangential velocity cannot develop properly. At this point the fluid goes straight to the outlet and no momentum transfer in tangential direction takes place. The turbine is flooded with the working fluid and stops the operation.

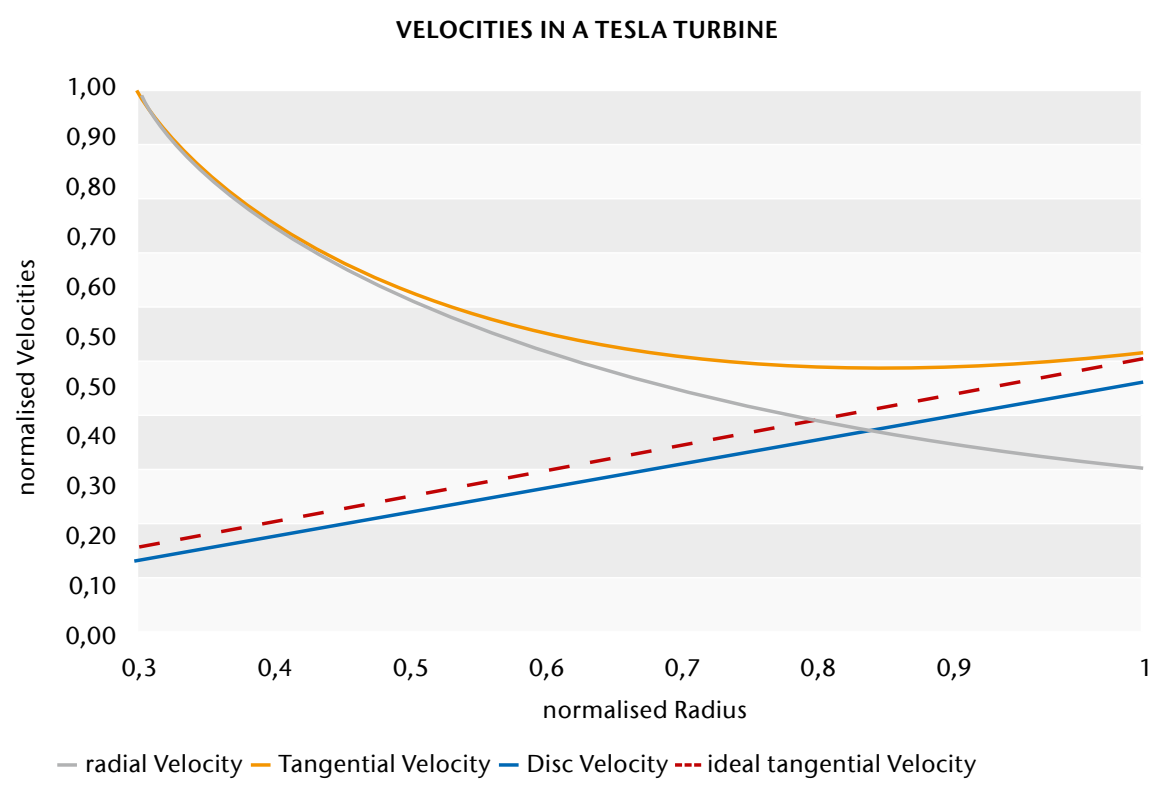

Fig. 2) Normalised velocities in a Tesla Turbine over the normalised radius. One is the outer perimeter and 0.3 the outlet.

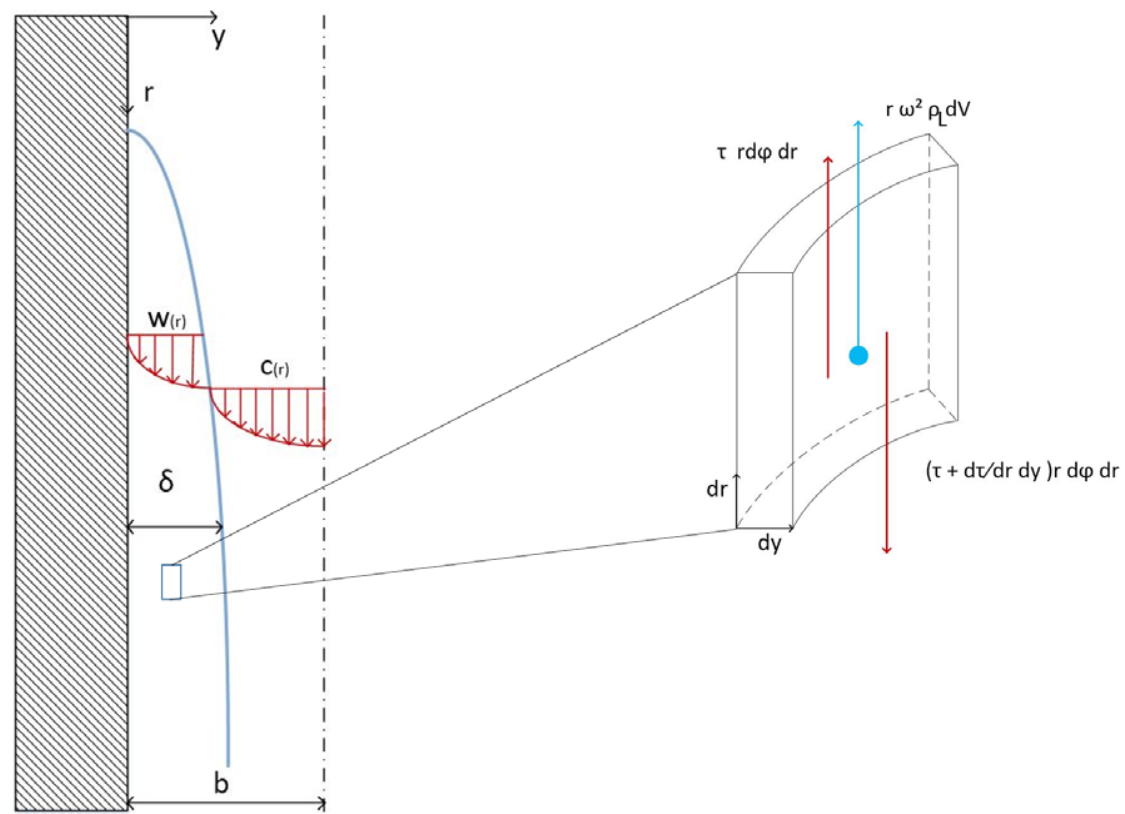

3 A simplified model for the development of a thin film in a Tesla turbine

In the following a new, simplified model for the establishment of a liquid thin film on a rotating disk is presented. The fluid film shall be very thin and present at the outlet region of the turbine so that a laminar film can be expected to develop. As mentioned before, the reason for film condensation is the reduction of the relative velocities towards the outlet by condensing the gaseous phase and decreasing the volume flow. The first closed theory on film condensation was presented by Nusselt (Stephan 1988). He calculated the thickness and the heat transfer in a water film on vertical plate under the influence of gravity. His findings were remarkable and showed a good agreement with experimental results. Later on these models have been refined and transferred to tubular and turbulent flows (Rose 1999). A similar model with condensation on a rotating plate was presented by Beckett, but here the flow of the working fluid goes from the centre to the outer perimeter (Beckett et al. 1973) which does not fit the flow field inside of the turbine.

However, in a Tesla turbine the fluid film flow in radial direction is governed by balance between the centrifugal forces and the applied surface drag from the gaseous working fluid. If the drag forces of the gas flow are higher than the centrifugal forces, the fluid film is pushed towards the outlet. If the centrifugal influence dominates the film starts spreading all over the disc. For the application in a Tesla turbine the fluid film has to be very thin due to the gap width. Otherwise the turbine can be clogged by the fluid film. To determine the film thickness, a force balance according to Fig. 3 has to be drawn. 
With the assumption of a parabolic the velocity profile in the film can be determined with the following equation.

$$
w(y)=-\frac{r \omega^{2} \rho_{L}}{\eta_{L}}\left(y^{2}-\delta y\right)+\frac{w_{\delta}(r)}{\delta} y
$$

Here $r$ is the radius, $\omega$ the rotational speed of the disk, $\rho_{L}$ the density of the liquid film, $\eta_{L}$ the dynamic viscosity of the film, $\delta$ the film thicknesses an $w_{\delta}$ the interfacial velocity. The velocity in the film can be depicted into the centrifugal force (1. Term of equation 4 ) and the interphase velocity $w_{\delta}$ at the phase boundary between the fluid and the gas flow.

The velocity profile of the gas flow can be determined with equation 5 . Here $c_{r}(r)$ is the radial velocity gained from equation $1 \mathrm{~b}$ the gap width and $c_{r \delta}$ is the velocity of the gas phase at the interface to the fluid film.

$$
c_{r(y)}=\frac{c_{r \delta}(r)-c_{r}(r)}{(\delta-b)^{2}}(y-b)^{2}+c_{r}(r)
$$

Within the system the no- slip condition applies, meaning that no slip between the wall, the fluid film and the gas phase occurs. This allows a momentum transfer between the single phases (wall - film and film - gas). Concluding from the no slip condition, it is apparent, that the velocity at the interfacial boundaries are equal. Therefore, on can substitute $c_{r \delta}(r)$ with $w_{\delta}(r)$ in equation 5 . Within equation 4 and 5 the interfacial velocity and the film thickness $\delta(\mathrm{r})$ are unknown. Under the assumption that both fluids are Newtonian fluids, the force balance at the phase boundary applies to

$$
\eta_{G} \frac{\partial w_{y=\delta}}{\partial y}=\eta_{L} \frac{\partial c_{r_{y=\delta}}}{\partial y}
$$

Solving equation 6 leads to the interfacial velocity $w_{\delta}$.

$$
\omega_{\delta}(r)=\frac{r \omega^{2} \rho_{L} \delta^{3}-r \omega^{2} \rho_{L} \delta^{2} b+2 c_{r}(r) \eta_{G} \delta}{2 \eta_{G} \delta+\eta_{L} b-\eta_{L} \delta}
$$

Combining equation 7 and equation 4 the velocity profile $w(y)$ of the fluid film can be determined. Further simplifications especially neglecting terms of higher order, lead to the velocity profile following profile.

$$
w(y)=\frac{r \omega^{2} \rho_{L} y^{2}}{\eta_{L}}-\frac{2 r \omega^{2} \rho_{L} \delta y}{\eta_{L}}+\frac{c_{r} 2 \eta_{G} y}{\eta_{L} b}
$$

With the velocity profile, the mass flow rate of the liquid film, the film thickness can be determined by the energy equation, which is in this case a comparison between the heat available at condensation temperature and the heat conducted (equation 9)

$$
\frac{\lambda_{L}}{\delta}\left(T_{s}-T_{w}\right) 2 \pi r d r=\Delta h_{v} d_{m}^{\phi} m
$$

Here $\Delta h_{v}$ is the enthalpy of vaporization and $\lambda_{L}$ is the thermal conductivity of the film. Rearranging and integrating of equation 9 leads to the final film thickness (equation 10).

$$
\delta(r)=\sqrt[3]{\frac{3\left(T_{s}-T_{w}\right) b v_{L} r}{\operatorname{Pr} T_{s}\left(2 \frac{\eta_{G}}{\eta_{L}} c_{r}(r)-R e_{\delta} \omega b\right)}}
$$

Here Pris the Prandl number for the liquid film and $R e_{\delta}$ is the Reynolds number. The later one will be low due to the thickness of the film. For the determination of the film thickness the denominator is of importance. Here the velocity between the fluid film and the gas flow is present. If $\operatorname{Re}_{\delta} \omega \mathrm{b}>2 \frac{\eta_{\mathrm{G}}}{\eta_{\mathrm{L}}} \mathrm{c}_{\mathrm{r}}(\mathrm{r})$ then the centrifugal forces are dominating and the film is pushed towards the inlet. Therefore, the other way around is preferred. From the theory above, we expect the film thickness to be in the range of $10-50 \mu \mathrm{m}$.

\section{Results}

For the calculation, it is assumed that the turbine is operated with steam/ water at a steady state and condensing takes place at the outlet region. The pressure in that region is $0,1 \mathrm{MPa}$ and the condensation temperature is $373,15 \mathrm{~K}$. From that on, the thermos physical properties of steam and water can be calculated. The turbine operates at $750 \mathrm{rpm}$ and the length of the film is assumed with $0,2 \mathrm{~m}$. The reason for choosing a steam/ water system are mostly utilisation reasons. For the following verification and testing of the presented model, the turbine test rig at the Technical University of Applied Sciences in Wildau, which is operated with water/steam, shall be used.

In the following picture left, the calculated film thickness for different rotation rates of the disk are presented. As one can see the film thickness is generally in the expected range. With a mass flow of $0.0018 \mathrm{~kg} / \mathrm{s}$ of steam, a disk rotation rate of $750 \mathrm{rpm}$ and an initial radial velocity of $35 \mathrm{~m} / \mathrm{s}$ a fluid film thickness of $26 \mu \mathrm{m}$ was achieved. Furthermore, the influence of the rotation rate $\omega$ is shown. Higher rotational rates do have an impact on the centrifugal forces, who pushes the mass of the film towards the inlet. The progression of the curve is bulgier at the start of the film resulting in a higher resistance for the gas flow.

From Fig. 4 left, one can further see that the film thickness decreases towards the outlet, which is caused by the increasing radial velocity. A higher radial velocity increases the drag on the film surface and therefore the film velocity increases which results in a thinner film. In Fig. 4 right, the interfacial velocity $W_{\delta}$ is presented for different mass flows. As mentioned above the interfacial velocity is of importance. A positive velocity indicates that the fluid film will develop and stay on the disk, since the drag 

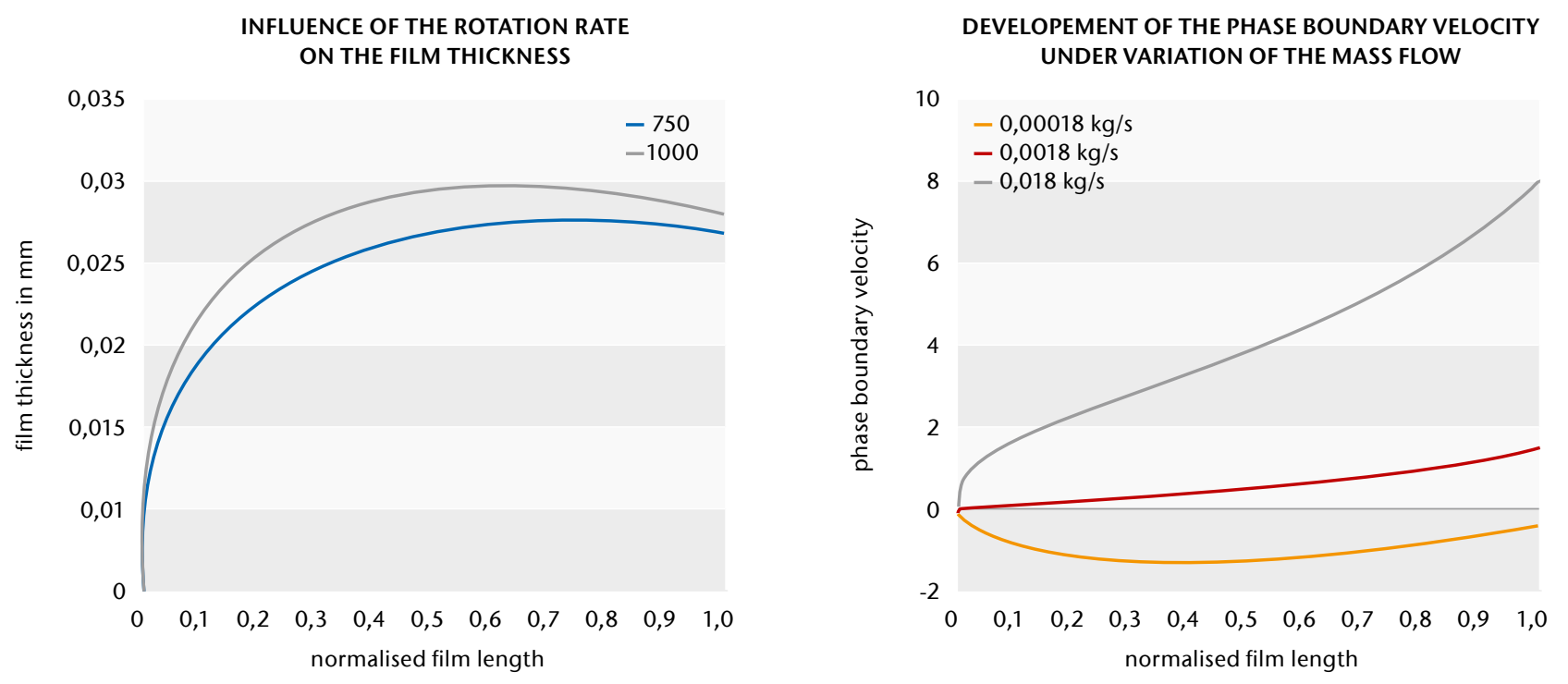

Fig. 4) Left: Calculated film thickness with a disk rotation rate of $7501 / \mathrm{s}$ and $100001 / \mathrm{s}$, Right: Interfacial velocity in dependence on the mass flow. Here 1 is the disk gap outlet and 0 is the beginning of the film development

REDUCTION OF THE GAS MASS FLOW

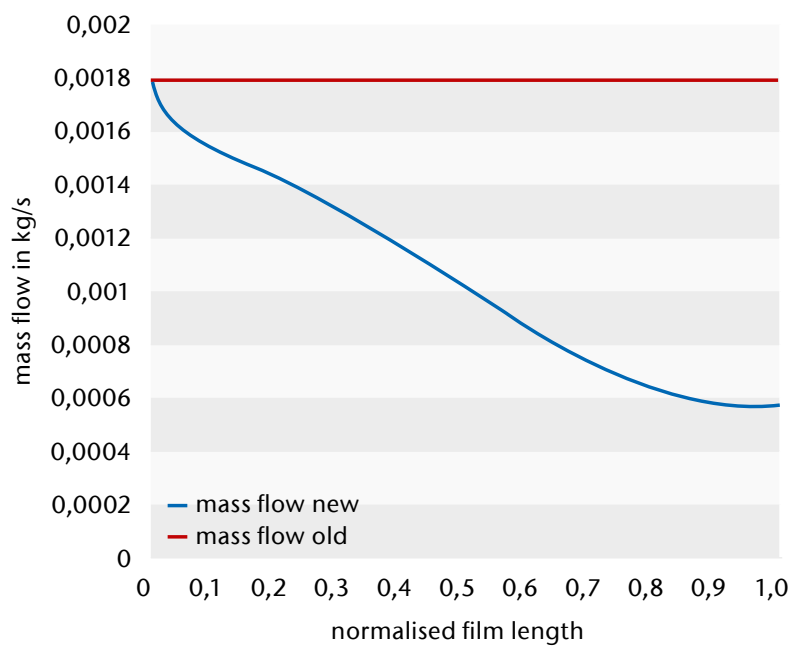

RADIAL VELOCITY WITH CONDENSATION

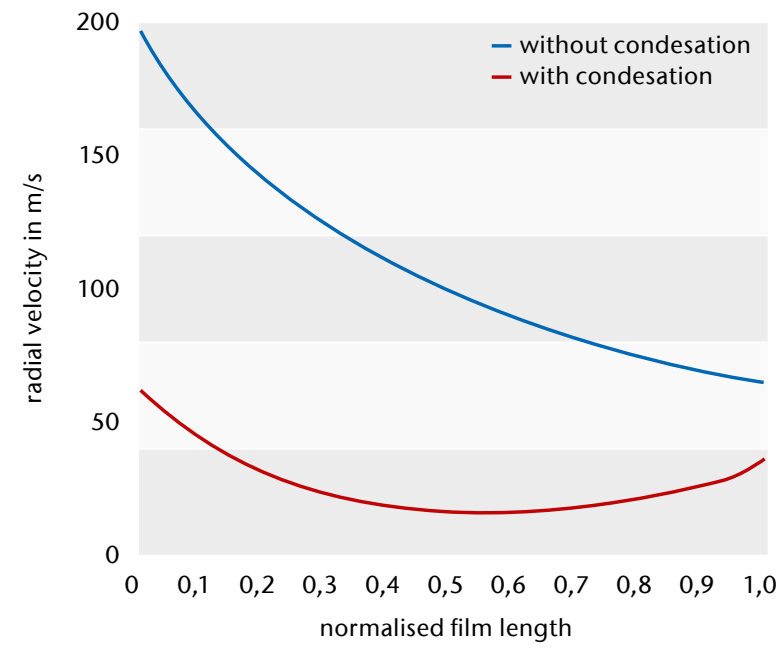

Fig. 5) Left: Adapted mass flow of the working fluid due to condensation, Right: Change in radial velocity due to condensation.

force applied by the radial velocity of the working fluid is higher than the centrifugal force induced by the disk rotation. A negative interfacial velocity shows that the centrifugal forces dominate and the film streams in the opposite direction. The grey line indicates a high mass flow, resulting into a high interfacial velocity, causing efficiency losses. The orange line represents an optimised interfacial velocity. The yellow line represents a negative interfacial velocity meaning that here the mass flow is too low to produce the necessary drag forces and establish a liquid film. Concluding one can say that the turbine operates best with low mass flow rates. Therefore, an op- timum has to be found for the establishment of a liquid thin film.

One goal of the fluid film is to reduce the radial velocities towards the outlet, since they account for efficiency losses. Per equation 1 the velocity is dependent on the mass flow of the working fluid and the cross- section area. By condensing the gaseous working fluid it states changes from gaseous to liquid, resulting in a lower specific volume and this results in a mass flow reduction of the gaseous phase. In Fig. 5 left, the reduction of the mass flow of the gaseous working fluid is shown. The red line indicates the mass flow without condensation and the blue line the mass flow with condensation.
Over the length of the fluid more than $50 \%$ of the gaseous working fluid will condensate which has a significant effect on the radial velocity. As shown in Fig. 5 right, the radial velocity decreases dramatically over the film length which shows that condensation at the outlet of a Tesla turbine decreases the velocity and therefore increases the turbine efficiency since the relative velocity between the disk and the working fluid is effectively lowered. 


\section{Discussion}

With the presented model, the utilisation of a laminar thin film at the outlet of a Tesla turbine gap element could have been successfully modelled. From the model the thickness of the fluid film could have been calculated -maximum depth of $26 \mu \mathrm{m}$-, which was in the expected range. Furthermore, the impact of the rotational speed of the disk on the film thickness and the importance of the radial gas velocity have been shown. It can be concluded that a higher radial velocity benefits the development of a laminar thin film due to the higher drag forces applied to the film. On the contrary, higher radial velocities are leading to efficiency losses. Therefore, like all engineering tasks, it is to find an optimum between drag forces and film thickness. To establish the fluid film, condensate must be present, which is generated by sub cooling the disks in the outlet area. With the ability to condensate inside of a Tesla turbine, the volume flow of the gaseous working fluid can be decreased dramatically, since the differences in specific volume between steam and water is in the range of 1 : 1640. Meaning that $1,64 \mathrm{~m}^{3}$ of steam at $0,1 \mathrm{MPa}$ and $100{ }^{\circ} \mathrm{C}$ is an equivalent of $0,001 \mathrm{~m}^{3}$ of water. Therefore, condensing has a big impact on lowering the specific volume and the radial and tangential velocity inside a Tesla turbine gap element. Form the model presented the adapted radial velocity was calculated. Assuming that there is no impact on the film thickness. This is wrong in a certain way. Since the change in radial velocity affects the film thickness and the interfacial velocity. In the next iteration, this change will be implemented. In general, one can state that as long as the interfacial velocity is bigger than $0 \mathrm{~m} / \mathrm{s}$ the drag applied to the film surface will push it towards the outlet.

Furthermore, the presented model is only valid for a laminar flow under the assumption that the tangential velocity of the gaseous working fluid has no impact on the centrifugal forces. This could be assumed since the film is very thin. But nevertheless, the tangential velocity will have an impact on the fluid film. Wave formation could occur, and therefore droplets could be pulled out of the film, resulting in a lower mass flow and depending on the droplet size, it could be pushed towards the inlet. In a practical application, the flow in radial direction must be present throughout the whole disk. This is not the case, since there are always minor changes in the radial velocity field (pressure drops from the nozzle, etc.). This has to be taken into account for the film design.

\section{Outlook}

With the presented model, the effects of film condensation in a disk turbine can be determined and described. By realising condensation inside of a disk turbine, the increasing velocities towards the outlet can be overcome and significantly reduced. The practical usage of a condensing turbine is huge. With such turbines, the steam cycle efficiency can be increased due to the improved enthalpy drop. The steam expansion can be ridden far more in the wet steam area, resulting into lower turbine outlet pressures and bigger energy extraction. With this advantage against conventional bladed turbines, the Tesla turbine could overcome its current winter sleep and face a future in industrial applications. At the moment, it is too early to predict an efficiency gain, since the theoretical model needs verification. But the results so far seem promising. Therefore, the next step is to develop a Tesla condensation turbine and verify the model, which will take place at the Turbine test rig at the Technical University of Applied Sciences in Wildau.

\section{REFERENCES}

Beckett PM, Hudson PC, Poots G (1973) Laminar film condensation due to a rotating disk. J Eng Math 7(1):63-73. doi: 10.1007/BF01535269

Guha A, Sengupta S (2013) The fluid dynamics of the rotating flow in a Tesla disc turbine. European Journal of Mechanics - B/Fluids 37:112-123. doi: 10.1016/j. euromechflu.2012.08.001

Holz C (2014) Ansätze zur serientechnischen Auslegung von Tesla-Turbinen. Bachelorarbeit, Technische Hochschule Wildau

Lisker R (2009) Numerische Strömungsuntersuchungen an einer Tesla Turbine. Diplomarbeit, Technische Hochschule Wildau

Lisker R (2013) The Design of a Tesla Turbine based Organic Rankine Cycle for Waste Heat Recovery. Master Thesis, Technische Hochschule Wildau

Lisker R, Hellwig U, Wildenauer FX (2015) Entwicklung und Leistungsbestimmung einer 2,2 kW Tesla Turbine. Wiss Beitr TH Wildau 19:61-66. doi: 10.15771/09498214_2015_1_8

Meller R (2013) Strömungstechnische Untersuchung der Eintritts- und Austrittsströmung an einer Scheibenturbine. Bachelorarbeit, Technische Hochschule Wildau Rose JW (1999) Condensation heat transfer. Heat and Mass Transfer 35(6):479-485. doi: 10.1007| s002310050351

Stephan K (1988) Wärmeübergang beim Kondensieren und beim Sieden. Wärme- und Stoffübertragung. Springer, Berlin, Heidelberg, New York, London, Paris, Tokyo. ISBN: 978-3-540-18075-3

Tesla N (1911) Turbine. Patent Application US1061206, 17 Jan 1911

\section{AUTHORS}

M.Eng. Roberto Liske

Prof. Dr.-Ing. Udo Hellwig

Prof. Dr. rer.nat. Franz Xaver Wildenaue

Technische Hochschule Wildau

E-Mail for correspondence:

roberto.lisker@th-wildau.de

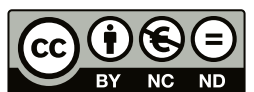

